\title{
Astrocytes rescue neuronal health after cisplatin treatment through mitochondrial transfer
}

Krystal English ${ }^{1}$, Andrew Shepherd ${ }^{1}$, Ndidi-Ese Uzor ${ }^{2,3}$, Ronnie Trinh ${ }^{1}$, Annemieke Kavelaars ${ }^{1}$ and Cobi J. Heijnen ${ }^{1 *}$ (D)

\begin{abstract}
Neurodegenerative disorders, including chemotherapy-induced cognitive impairment, are associated with neuronal mitochondrial dysfunction. Cisplatin, a commonly used chemotherapeutic, induces neuronal mitochondrial dysfunction in vivo and in vitro. Astrocytes are key players in supporting neuronal development, synaptogenesis, axonal growth, metabolism and, potentially mitochondrial health. We tested the hypothesis that astrocytes transfer healthy mitochondria to neurons after cisplatin treatment to restore neuronal health.

We used an in vitro system in which astrocytes containing mito-mCherry-labeled mitochondria were co-cultured with primary cortical neurons damaged by cisplatin. Culture of primary cortical neurons with cisplatin reduced neuronal survival and depolarized neuronal mitochondrial membrane potential. Cisplatin induced abnormalities in neuronal calcium dynamics that were characterized by increased resting calcium levels, reduced calcium responses to stimulation with $\mathrm{KCl}$, and slower calcium clearance. The same dose of cisplatin that caused neuronal damage did not affect astrocyte survival or astrocytic mitochondrial respiration. Co-culture of cisplatin-treated neurons with astrocytes increased neuronal survival, restored neuronal mitochondrial membrane potential, and normalized neuronal calcium dynamics especially in neurons that had received mitochondria from astrocytes which underlines the importance of mitochondrial transfer. These beneficial effects of astrocytes were associated with transfer of mitochondria from astrocytes to cisplatin-treated neurons. We show that siRNA-mediated knockdown of the RhoGTPase Miro-1 in astrocytes reduced mitochondrial transfer from astrocytes to neurons and prevented the normalization of neuronal calcium dynamics.

In conclusion, we showed that transfer of mitochondria from astrocytes to neurons rescues neurons from the damage induced by cisplatin treatment. Astrocytes are far more resistant to cisplatin than cortical neurons. We propose that transfer of functional mitochondria from astrocytes to neurons is an important repair mechanism to protect the vulnerable cortical neurons against the toxic effects of cisplatin.
\end{abstract}

Keywords: Astrocytes, Cortical neurons, Mitochondria, Cisplatin, Miro-1

\section{Significance statement}

Chemotherapy-induced neurotoxicity is a serious health problem and little is known about the underlying mechanisms. Especially neurons are very sensitive to cisplatin treatment while astrocytes are not. We show that astrocytes can protect neurons damaged by cisplatin by

\footnotetext{
* Correspondence: Cjheijnen@mdanderson.org

'Division of Internal Medicine, Department of Symptom Research,

Laboratories of Neuroimmunology, The University of Texas MD Anderson

Cancer Center, Houston, TX 77030, USA

Full list of author information is available at the end of the article
}

improving neuronal survival, mitochondrial health, and calcium dynamics in vitro. This beneficial effect of astrocytes is dependent on the transfer of mitochondria from astrocytes to the damaged neurons. Our findings provide evidence for an important endogenous protective neuroglial mechanism that could contribute to prevention of neuronal death as a result of cisplatin treatment and thereby aid in sustaining brain health of patients during chemotherapy.

(c) The Author(s). 2020 Open Access This article is distributed under the terms of the Creative Commons Attribution 4.0 International License (http://creativecommons.org/licenses/by/4.0/), which permits unrestricted use, distribution, and 


\section{Introduction}

Mitochondria are unique organelles that are crucial for sustaining cellular health through multiple functions, including ATP production via oxidative phosphorylation, metabolic regulation, regulation of apoptosis, and $\mathrm{Ca}^{2+}$ buffering [1-3]. Neurons are highly specialized cells that, like all cells, are critically dependent on intact mitochondrial function for rapidly responding to changes in energy demand, storing and buffering $\mathrm{Ca}^{2+}$ and, specifically for neurons, neurotransmission and plasticity [4-7]. Due to the critical importance of mitochondria for multiple key aspects of neuronal function, it is not surprising that mitochondrial dysfunction can have devastating effects on brain function [8-10].

Although cisplatin treatment of neurons in vitro or ex vivo leads to cell death, adult neurons do not massively die after cisplatin treatment in vivo suggesting there is an endogenous protective mechanism in place [8]. However, long lasting cisplatin treatment leads to neuronal mitochondrial dysfunction, which has severe consequences for brain function including cognition $[8,11]$.

Astrocytes release multiple factors that are essential to neuronal development, signaling, metabolism, axonal growth and synaptogenesis [12-16]. Recent evidence indicates an additional way via which astrocytes can contribute to neuronal health is by donating healthy mitochondria to damaged neurons $[17,18]$. Specifically, Wang et al. showed that exposure of rat hippocampal astrocytes and neurons to $\mathrm{H}_{2} \mathrm{O}_{2}$ or serum deprivation promotes transfer of mitochondria from astrocytes to neurons. Moreover, astrocytes not only function as donor of healthy mitochondria but can also function as recipient of damaged mitochondria. Davis et al. showed that retinal ganglion cell axons routinely shed mitochondria at the optic nerve head to be degraded by astrocytes in vivo. Mitochondrial transfer from one cell type to another is not exclusive to astrocytes and neurons [19]. For example, we and others have shown that mesenchymal stem cells transfer mitochondria to damaged neuronal stem cells thereby improving stem cell survival and mitochondrial membrane potential in the recipient cells [20-22]. Transfer of mitochondria from one cell to another occurs via multiple mechanisms such as release and uptake of vesicles, transfer via gap junctions, and transfer via F-actin based tunneling nanotubes [17, 23]. Mitochondrial RhoGTPase 1 (Miro-1) is a calcium-sensitive adaptor protein that drives movement of mitochondria along microtubules [24-28]. Miro-1 is involved in transferring mitochondria from mesenchymal stem cells to neuronal stem cells [20, 29], but its contribution to mitochondrial transfer from astrocytes to neurons is unknown.

Multiple neurodegenerative disorders, including Parkinson's disease, Alzheimer's disease, and chemotherapyinduced cognitive impairment are associated with neuronal mitochondrial dysfunction [2, 8-10, 30-32]. We have shown recently that treatment of mice with the chemotherapeutic drug cisplatin results in synaptosomal mitochondrial dysfunction that causes cognitive deficits [8]. Cisplatin crosses the blood-brain barrier at levels that are sufficient to cause damage to hippocampal neurons and to neuronal stem cells [33]. However, cisplatin treatment in vivo does not lead to overt neuronal cell death which could indicate that there are endogenous protective mechanisms, such as mitochondrial transfer by astrocytes, to assist in sustaining neuronal health in conditions of acute danger to adult neurons.

The aim of the current study is to test the hypothesis that astrocytes transfer mitochondria to neurons damaged by cisplatin and thereby improve neuronal function and health in vitro.

\section{Materials and methods \\ Culture of cortical neuron and astrocytes}

Timed-pregnant Long Evans rats (Charles River, Wilmington, MA, USA) were sacrificed and E18 fetuses of both sexes were collected in accordance with Institutional Animal Care and Use Committee-approved protocols.

Cortices were dissected and incubated in $10 \mathrm{ml}$ of dissociation media $\left(81.8 \mathrm{mM} \mathrm{Na}_{2} \mathrm{So}_{4}, 30 \mathrm{mM} \mathrm{K} \mathrm{SO}_{4}, 5.8\right.$ $\mathrm{mM} \mathrm{MgCl}_{2}, 0.25 \mathrm{mM} \mathrm{CaCl}_{2}, 1 \mathrm{mM}$ Hepes, $20 \mathrm{mM}$ glucose, $0.0001 \%$ Phenol Red, $0.16 \mathrm{mM} \mathrm{NaOH} \mathrm{pH}=7.4$ ) that contained $10 \mathrm{U} / \mathrm{mL}$ papain and $5 \mathrm{mg}$ of L-cysteine in total (Worthington, Lakewood, NJ, USA) for $10 \mathrm{~min}$ at $37^{\circ} \mathrm{C}$, followed by incubation with $150 \mathrm{mg}$ of trypsin inhibitor (Millipore-Sigma, St. Louis, MO) in $10 \mathrm{ml}$ of dissociation media for $10 \mathrm{~min}$ at $37^{\circ} \mathrm{C}$. The cortical tissue was mechanically dissociation in Opti-mem (GIBCO, Carlsbad, CA, USA) with $2.5 \mathrm{M}$ Glucose (GIBCO). Cells were cultured on plates coated with $0.05 \mathrm{mg} / \mathrm{ml}$ poly-Dlysine (PDL; Millipore-Sigma) in neurobasal medium (NBM) with $100 \mathrm{U} / \mathrm{mL}$ penicillin and $1 \mathrm{x}$ B-27 supplement (Invitrogen Carlsbad, CA) at $37^{\circ} \mathrm{C}$ and $5 \% \mathrm{CO}_{2}$. Neuronal cultures were maintained in NBM with B-27 supplement and media was replaced every 3 days. Cortical astrocytes were grown in DMEM/F12 medium supplemented with $10 \%$ fetal bovine serum and $5 \%$ of 10 , 000 units / $\mathrm{ml}$ of penicillin and $10,0000 \mu \mathrm{g} / \mathrm{ml}$ of streptomycin (GIBCO) at $5 \% \mathrm{CO}_{2}$ and $37^{\circ} \mathrm{C}$.

Neuronal cells were used for experiments after 12-15 days of culturing in vitro (DIV). To confirm neuronal enrichment, cells were fixed with $4 \%$ paraformaldehyde in PBS, treated with $0.25 \%$ Triton X-100, blocked in $2 \%$ BSA in PBS and stained with anti-Map2 antibody (1: 2000); Sigma-Aldrich); anti-GFAP (1:200, Acris, Rockville, MD) and anti-Olig2 (1:400, Abcam, Cambridge, UK) antibody. On DIV12 > 98\% of cells were Map2+ and Olig2 and GFAP-negative. Astrocytes were used until the third passage and were $>99 \%$ GFAP+. 


\section{Astrocyte transfections}

Astrocytes were plated in a 6-well plate at $1.5 \times 10^{5}$ cells/ well $24 \mathrm{~h}$ before transfection. For labeling mitochondria, astrocytes were transfected with $2 \mu \mathrm{g}$ of pLYS1-FLAGMitoGFP-HA (Addgene plasmid \# 50057) which contains the pore-forming subunit of the mitochondrial calcium uniporter coupled to GFP or a mito-mCherry construct generated by subcloning the targeting sequence of the pLYS1-FLAG-MitoGFP-HA plasmid into the mcherry2N1 vector (Addgene plasmid \# 54517). For Miro-1 knockdown, $5 \mathrm{nmol}$ of Rho-1 siRNA (Qiagen, Germany \#SI01401743) was diluted in rnase-free water (provided in kit) to make a $20 \mu \mathrm{M}$ stock, andAllStars Negative Control Scrambled siRNA (Qiagen \#SI03650318) was performed similarly to make a $20 \mu \mathrm{M}$ stock instructions provided. Astrocytes were transfected with $80 \mathrm{nM}$ of Rho-1 siRNA from a $20 \mu \mathrm{M}$ stock (Qiagen, Germany \#SI01401743) or 80 nM AllStars Negative Control Scrambled siRNA from a $20 \mu \mathrm{M}$ stock (Qiagen \#SI03650318). All transfections were performed using the Astrocyte Transfection kit (Altogen Biosystems, Las Vegas, NV, USA) according to manufacturer's instructions. Miro-1 knockdown was confirmed by Western blot with anti-Rho1 antibody (Novus Biologicals, Centennial, CO, USA) with GAPDH as control (Abcam) followed detection of bands with enhanced chemoluminescence (GE Healthcare Bio-Sciences, Pittsburgh, PA). Blots were captured in the LAS system using Image Quant software (GE Healthcare Bio-Sciences) for quantification of bands.

\section{Analysis of neuronal survival}

Neurons were plated in 96 well plates coated with 0.05 $\mathrm{mg} / \mathrm{ml} \mathrm{PDL}$ at $5 \times 10^{4}$ neurons/well. Viability after exposure to cisplatin (Teva, Petah Tikva, Israel) was quantified using the colorimetric cell viability reagent WST-1 (Millipore-Sigma, \#11644807001). To assess the effect of astrocytes on neuronal survival, separate cultures of neurons $\left(1.5 \times 10^{5}\right.$ cells/well in a 6-well plate) and astrocytes $\left(5 \times 10^{4} /\right.$ well in a 6 -well plate $)$ were treated with cisplatin for $24 \mathrm{~h}$. Neurons were then labeled with $20 \mu \mathrm{M}$ CellTracker Blue (CTB; Invitrogen) for $45 \mathrm{~min}$ at $37^{\circ} \mathrm{C}$, and washed in serum-free media. Astrocytes $\left(5 \times 10^{4}\right.$ cells/well) were added to the neuronal culture and survival of $\mathrm{CTB}+$ neurons was quantified $17 \mathrm{~h}$ later using a Countess II FL automated cell counter (Invitrogen).

\section{Analysis of mitochondrial membrane potential and mitochondrial transfer}

Neurons were plated at $1.5 \times 10^{5}$ cells/well in a 6-well plate, treated with cisplatin for $24 \mathrm{~h}$, and labeled with $20 \mu \mathrm{M}$ CellTracker Green (Thermo Fisher). Astrocytes $\left(5 \times 10^{4}\right.$ cells/well $)$ were added and co-cultured with the neurons for $17 \mathrm{~h}$. The co-cultures were stained with tetramethylrhodamine methyl ester (TMRM, Invitrogen; 250
$\mathrm{nM})$ for $45 \mathrm{~min}$ at $37^{\circ} \mathrm{C}$, or Mitotracker $(50 \mathrm{nM}$, Thermo Fisher, Waltham, MA, USA) for $30 \mathrm{~min}$ at $37^{\circ} \mathrm{C}$. As a positive control, neurons were treated with $10 \mu \mathrm{M}$ carbonilcyanide p-triflouromethoxyphenylhydrazone (FCCP, SigmaAldrich), a mitochondrial uncoupler, for $15 \mathrm{~min}$. Cells were collected and TMRM fluorescence intensity of the cell tracker green positive cells was quantified using an Accuri C6 Flow Cytometer (BD Biosciences, San Jose, CA USA). For confocal microscopy, neurons were plated in cell culture imaging dishes (ibidi, Fitchburg, WI, USA), treated with cisplatin, stained with CTB and cultured with or without mito-GFP and cell tracker deep red-labeled astrocytes for $17 \mathrm{~h}$ followed by staining with TMRM or Mitotracker. The TMRM was used in sub-quench mode as described previously [34].

For analysis of mitochondrial transfer, neurons exposed to cisplatin or vehicle were labeled with $20 \mu \mathrm{M}$ CTB (Thermo Fisher) prior to co-culture with astrocytes. Astrocytes were transfected with either mito-GFP or mitomCherry prior to exposure to cisplatin followed by labeling with $20 \mu \mathrm{M}$ Celltracker DeepRed (Thermo Fisher) and co-culture with neurons. Neurons containing an area positive for the mito-GFP or mito-mCherry signal that was larger than 8 pixels were scored as containing astrocytederived mitohcondria. The co-cultures were imaged on an SPE Leica Confocal Microscope (Leica Microsystems, Buffalo Grove, IL, USA) with a $63 \mathrm{X}$ or $40 \mathrm{X}$ objective and images were analyzed with LAS X software.

\section{Analysis of mitochondrial bioenergetics}

To assess mitochondrial bioenergetics, astrocytes $(5 \times$ $10^{4}$ cells/well) were plated in a Seahorse XFe 24 microplate (Seahorse Biosciences/Agilent Technologies, Santa Clara, CA, USA) coated with $0.05 \mathrm{mg} / \mathrm{ml} \mathrm{PDL} \mathrm{and}$ treated with $1 \mu \mathrm{M}$ cisplatin or vehicle for $24 \mathrm{~h}$. Cells were washed and incubated for $1 \mathrm{~h}$ at $37^{\circ} \mathrm{C}$ in XF base media (Seahorse Biosciences) supplemented with $11 \mathrm{mM}$ glucose (Sigma-Aldrich), $2 \mathrm{mM}$ glutamine (Sigma Aldrich), and $1 \mathrm{mM}$ pyruvate (Sigma-Aldrich), $2 \mathrm{mM}$ Oligomycin (Sigma-Alrich), $4 \mathrm{mM} \mathrm{FCCP,} \mathrm{and} \mathrm{rotenone/}$ antimycin A (Sigma-Aldrich, $2 \mathrm{mM}$ each) were used with a 3-time repeat of a 2-min mix, 3-min wait, and 2-min measure assay cycle. Oxygen consumption rates were normalized to the total protein content of each well. Basal respiration, maximal respiratory capacity, and spare respiratory capacity were determined as described previously [20].

\section{Calcium imaging}

Functional $\mathrm{Ca}^{2+}$ imaging on cortical neurons was performed as described previously [35]. $12 \mathrm{~mm}$ circular glass coverslips containing cells were incubated at room temperature for $20 \mathrm{~min}$ with the $\mathrm{Ca}^{2+}$-sensitive dye Fura-2AM (Invitrogen, $2 \mu \mathrm{M}$ ), dissolved in standard extracellular 
HEPES-buffered HBSS (known hereafter as extracellular imaging buffer) containing the following (in $\mathrm{mM}$ ): 140 $\mathrm{NaCl}, 5 \mathrm{KCl}, 1.3 \mathrm{CaCl}_{2}, 0.4 \mathrm{MgSO}_{4}, 0.5 \mathrm{MgCl}_{2}, 0.4$ $\mathrm{KH}_{2} \mathrm{PO}_{4}, 0.6 \mathrm{NaHPO}_{4}, 3 \mathrm{NaHCO}_{3}, 10$ glucose, and 10 HEPES adjusted to pH 7.4 with $\mathrm{NaOH}$ and 310 mOsm with sucrose. The coverslip was placed in the recording chamber (ALA scientific Instruments, Farmingdale, NY, USA) mounted on the stage of an inverted Nikon Ti2 microscope and continuously superfused for $5 \mathrm{~min}$ at room temperature with extracellular imaging buffer. Fura-2 fluorescence was alternately excited at 340 and $380 \mathrm{~nm}$ (12 $\mathrm{nm}$ band-pass, $50 \mathrm{~ms}$ exposure) at $1 \mathrm{~Hz}$ using a Lambda LS Xenon lamp (Sutter Instruments, Novato, CA, USA) and a 10x/NA 0.5 objective or 40x/NA 0.6. The CFI Super Fluor $10 \mathrm{X}$ was used to measure the ratio of Fura-2 fluorescence. Emitted fluorescence was collected at $510 \mathrm{~nm}$ using a sCMOS pco.edge camera for the entire experimental duration, including the first $5 \mathrm{~min}$ wash duration, and the ratio of fluorescence (F340:F380) was calculated. The shift in the ratio of Fura-2 fluorescence between excitation at $340 \mathrm{~nm}$ versus $380 \mathrm{~nm}$ is used as a readout of changes in intracellular calcium concentration $\left[\mathrm{Ca}^{2+}{ }_{i}\right]$. Baseline recording of F340:F380 ratio without stimulus was completed to obtain an indication of resting $\left[\mathrm{Ca}^{2+}{ }_{\mathrm{i}}\right]$. Neurons were stimulated with $20 \mathrm{mM}$ of $\mathrm{KCl}$ in the extracellular imaging buffer with continuous superfusion at room temperature. Imaging was analyzed with the NIS Elements software. To identify neurons that did or did not receive astrocytic mitochondria, the Nikon inverted Ti2 microscope with associated A1RsiHD confocal system and NIS Elements software was used with the CFI S Plan Fluor ELWD 40XC objective. A single exposure at $576 \mathrm{~nm}$ excitation was used to quantify fluorescence within neurons before beginning measurement of F340:F380 ratio.

\section{Data analysis}

Data are presented as mean \pm SEM of at least 3 independent experiments performed in duplicate or triplicate. For survival analysis and mitochondrial membrane potential analysis, data were normalized to the control of each experiment and replicates were averaged. We used One-way or Two-way analysis of variance (ANOVA) with or without repeated measure followed by Tukey's correction for multiple comparisons or Sidak's correction for multiple comparisons according to experimental set up. For mitochondrial transfer analysis, the percentage of neurons that received mitochondria was calculate for each group. We used Student's t-test or Two-way analysis of variance (ANOVA) without repeated measures followed by Tukey's correction for multiple comparisons. For calcium imaging, the ratio of fluorescence (F340:F380) was calculated and the ratios at baseline were subtracted from the maximum peak values upon $\mathrm{KCl}$ stimulus to calculate change in $\left[\mathrm{Ca}^{2+}{ }_{\mathrm{i}}\right]$. To calculate $\mathrm{Ca}^{2+}$ clearance, F340:F380 ratios were converted to a 0-1 scale with 1 being the maximum value in response to $20 \mathrm{mM} \mathrm{KCl}$. Then, we quantified the time to return to $20 \%$ of baseline by counting the values from 1 (maximum peak) to 20\% of the baseline value for each neurons. All analyses were performed using GraphPad Prism 8 (GraphPad Software, La Jolla, CA, USA).

\section{Results}

\section{Astrocytes improve neuronal survival after cisplatin treatment}

To examine the effect of cisplatin on neuron and astrocyte survival, we treated separate cultures of primary cortical neurons and astrocytes with cisplatin for $24 \mathrm{~h}$ and measured survival using the WST assay. Cisplatin dose-dependently reduced neuronal survival as assessed immediately after removal of cisplatin (Fig. 1a) or $17 \mathrm{~h}$ later (Fig. 1b). Astrocytes were shown to be resistant to cisplatin since only the highest dose $(4 \mu \mathrm{M})$ resulted in a modest reduction in astrocyte survival (Fig. 1c and d). For all further experiments we used cisplatin at a dose of $1 \mu \mathrm{M}$. Exposure of astrocytes to $1 \mu \mathrm{M}$ cisplatin did not induce detectable changes in astrocytic mitochondrial respiration; basal respiration, spare respiratory capacity and maximal respiration were similar in control and cisplatin-treated astrocytes (Fig. 1e).

To test the hypothesis that astrocytes improve the survival of neurons treated with cisplatin, primary cortical neurons and astrocytes were cultured separately in the presence or absence of $1 \mu \mathrm{M}$ cisplatin for $24 \mathrm{~h}$. Next cisplatin was removed, neurons were labeled with celltracker blue (CTB), and co-cultured with astrocytes for an additional $17 \mathrm{~h}$. Co-culture with astrocytes significantly improved survival of cisplatin-treated neurons. Astrocytes did not affect survival of control neurons (Fig. 1f).

\section{Astrocytes improve mitochondrial membrane potential of neurons damaged by cisplatin}

To assess the effect of cisplatin on neuronal mitochondrial integrity, we quantified mitochondrial membrane potential by labeling the cells with tetramethylrhodamine (TMRM) and assessed fluorescence intensity by flow cytometry. Figure 2a clearly shows that cisplatin depolarized the neuronal mitochondrial membrane potential as indicated by a reduction in TMRM fluorescence intensity. The cisplatin-induced reduction in TMRM fluorescence intensity was not associated with changes in neuronal mitochondrial content as determined by labeling mitochondria with the membrane potential-independent dye Mitotracker (Fig. 2b). Together these findings indicate that cisplatin reduces neuronal mitochondrial membrane potential without leading to an actual loss of mitochondria under the conditions tested. Co-culture with astrocytes restored the mitochondrial membrane potential of neurons 

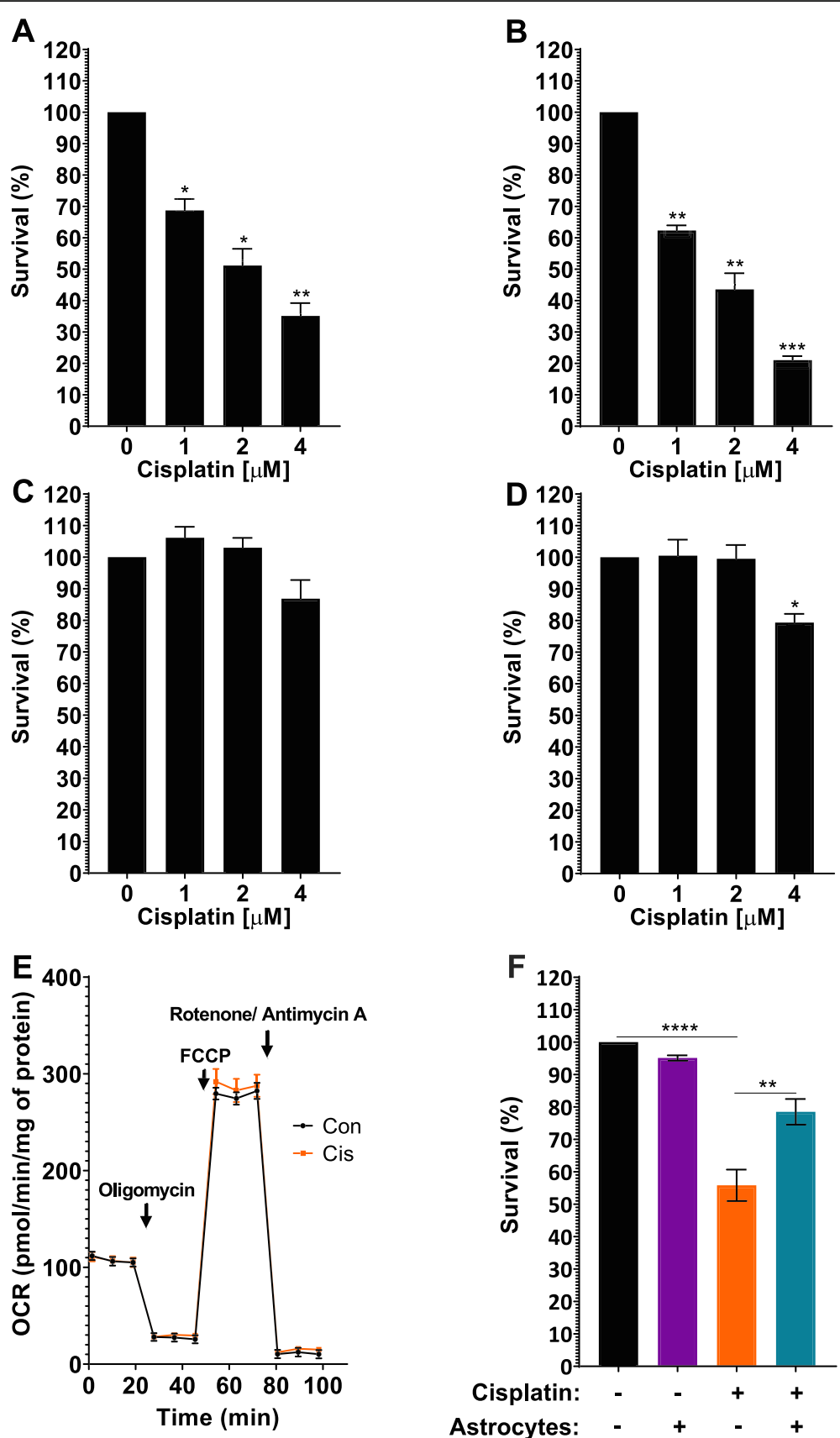

Fig. 1 Astrocytes improve survival of neurons damaged by cisplatin. a-d. Survival of neurons $(\mathbf{a}, \mathbf{b})$ and astrocytes (c, d) after exposure to cisplatin. Primary cultures of cortical neurons (12 days in vitro) or cortical astrocytes were treated with increasing doses of cisplatin or vehicle for $24 \mathrm{~h}$, then the cisplatin was removed and replaced with media. Survival was measured using a WST-1 assay: (a, c) at the end of the $24 \mathrm{~h}$ culture period or (b, d) $17 \mathrm{~h}$ after removal of cisplatin. Data was normalized to survival in the absence of cisplatin and analyzed using one-way ANOVA followed by Dunnett's multiple comparisons test $\left({ }^{*} p<0.05,{ }^{* *} p<0.01,{ }^{* * *} p<0.001\right.$ ). e. Astrocytes were treated with $1 \mu \mathrm{M}$ cisplatin for $24 \mathrm{~h}$ followed by $17 \mathrm{~h}$ of culture without cisplatin. Oxygen consumption rates (OCR) were analyzed using Seahorse XFe 24 Analyzer and normalized to protein content. $\mathbf{f}$. Neurons and astrocytes were treated with cisplatin or control medium for $24 \mathrm{~h}$. Cisplatin was removed, neurons were labeled with Celltracker Blue (CTB) and cultured for another $17 \mathrm{~h}$ with or without astrocytes, and the number of surviving neurons was counted. Data are normalized to control and represents the mean \pm SEM of 3 independent experiments performed in duplicate. Two-way ANOVA: cisplatin $x$ astrocyte interaction: ${ }^{* *} p<0.01$; Tukey's multiple comparisons post hoc test $\left({ }^{*} p<0.05,{ }^{* *} p<0.01,{ }^{* * * *} p<0.0001\right)$ 

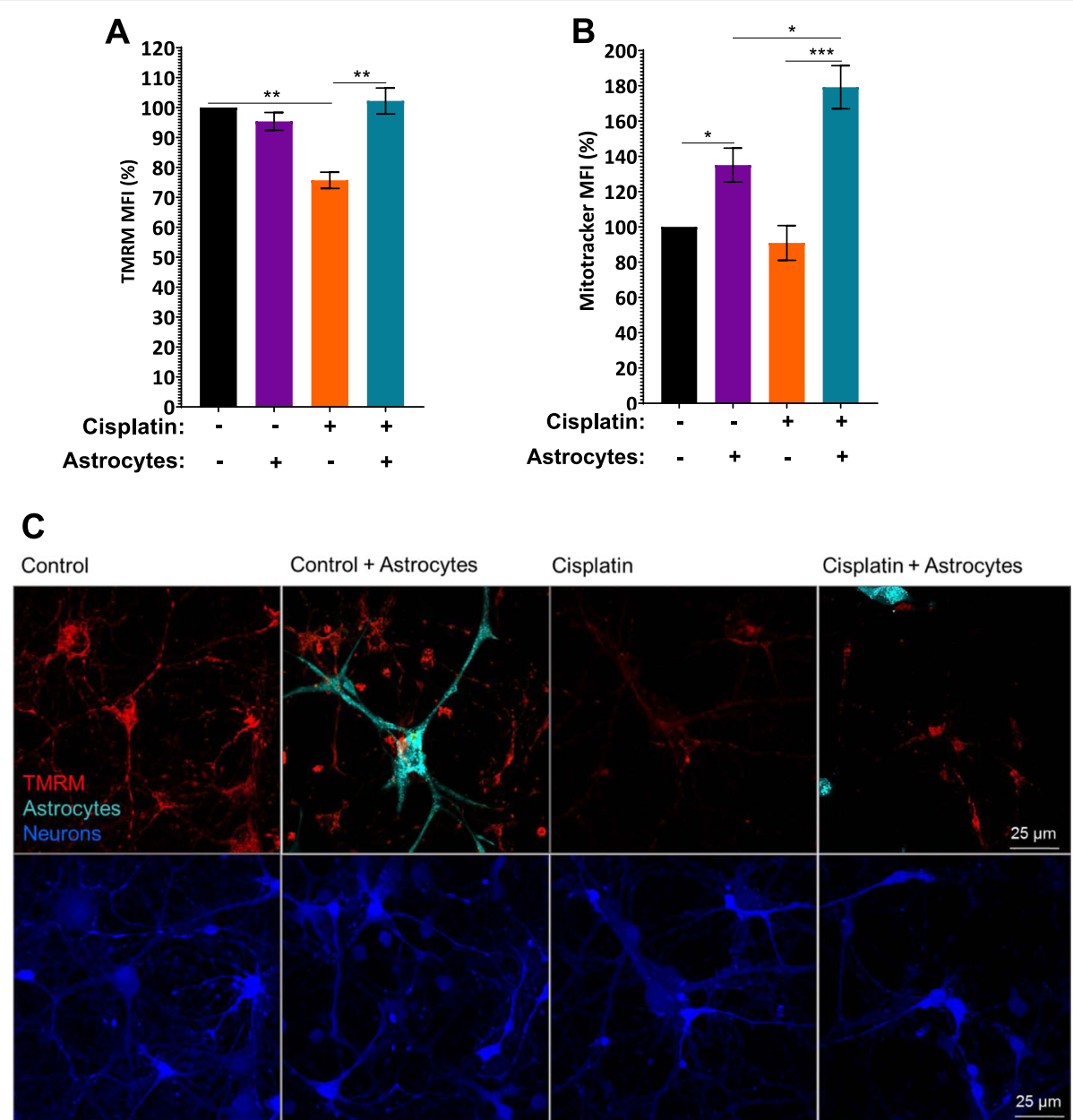

Fig. 2 Astrocytes improve mitochondrial membrane potential of neurons damaged by cisplatin. After treating neurons and astrocytes separately with and without cisplatin for $24 \mathrm{~h}$, neurons were labeled with Celltracker Green (CTG) and co-cultured with astrocytes for $17 \mathrm{~h}$. Cells were labeled with TMRM (a) to assess mitochondrial membrane potential or with mitotracker (b) to quantify mitochondrial content by FACS analysis.

Fluorescence intensity was normalized to fluorescence intensity in control CTG-positive neurons for each experiment and data represents the mean \pm SEM of 3 independent experiments performed in duplicate. Two-way ANOVA TMRM: cisplatin $x$ astrocyte interaction: ${ }^{*} p<0.05$; Tukey's post-hoc test: ${ }^{* *} p<0.01$. Mitotracker: cisplatin $x$ astrocyte interaction: ${ }^{*} p<0.05$; Tukey post-test: ${ }^{*} p<0.05$, ${ }^{* * *} p<0.001$. c. Representative confocal images of the TMRM signal (red) with neurons labeled with CTB and astrocytes with cell tracker deep red (Teal). Scale bar: $25 \mu \mathrm{m}$

cultured with cisplatin (Fig. 2a). Confocal microscopy confirmed that cisplatin reduces TMRM staining in somata and dendrites of neurons and that co-culture with astrocytes restored TMRM staining (Fig. 2c). The increase in TMRM fluorescence intensity of cisplatintreated neurons in response to astrocytes was associated with an increase in mitotracker fluorescence intensity in neuronal cells (Fig. 2b), indicating an increase in neuronal mitochondrial content as a result of co-culture with astrocytes.

\section{Astrocytes transfer mitochondria to neurons damaged by cisplatin}

Next, we tested the hypothesis that astrocytes transfer mitochondria to neurons damaged by cisplatin. We labeled astrocyte mitochondria with mCherry coupled to a mitochondrial localization sequence (mito-mCherry). Neurons and astrocytes were treated separately with cisplatin or control medium for $24 \mathrm{~h}$; neurons were labeled with Celltracker Blue, and co-cultured with Celltracker Deep Redlabeled astrocytes for an additional $17 \mathrm{~h}$. Confocal fluorescence analysis revealed that cisplatin-treated neurons contained astrocyte-derived mCherry+ mitochondria. Only a few astrocyte-derived mitochondria were present in untreated control neurons co-cultured with astrocytes (Fig. 3ac). These findings indicate that astrocytes transfer mitochondria to neurons damaged by cisplatin. Quantitative assessment of mitochondrial transfer showed that cisplatin induced an approximately 3 -fold increase in the percentage of neurons that had received mitochondria from astrocytes (Fig. 3d). 3D reconstruction and orthogonal section analysis confirmed that the mito-mCherry+ mitochondria are 

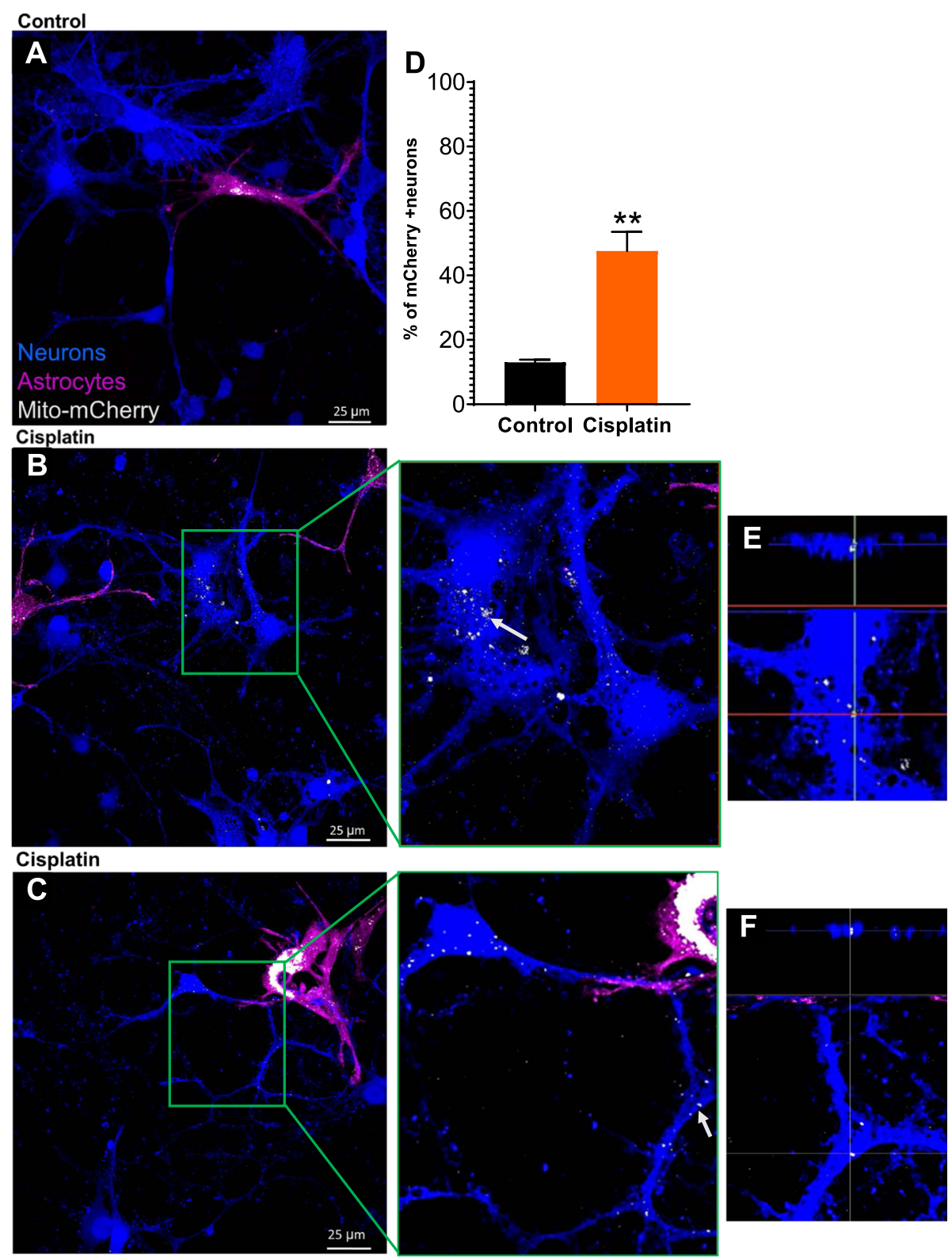

Fig. 3 Astrocytes transfer mitochondria to neurons damaged by cisplatin. a. Representative confocal image of untreated neurons co-cultured for $17 \mathrm{~h}$ with astrocytes in which mitochondria were labeled with mCherry. b-c. Representative confocal images of co-cultures of cisplatin-treated neurons labeled with CTB and cisplatin-treated astrocytes in which mitochondria were labeled with mCherry. Middle panel: larger magnification of the boxed area in B/C. e-f. 3-D reconstruction and orthogonal slicing showing that the astrocyte-derived mCherry-positive mitochondria (identified by arrows in the middle panel) are present within the neurons. Scale bar: $25 \mu \mathrm{m}$. $\mathbf{d}$. Quantification of neurons in co-cultures containing $m$ Cherry+ astrocyte-derived mitochondria. Paired Student's t-test ${ }^{* *} p<0.01$. Data represents the mean \pm SEM of 3 independent experiments performed in duplicate

localized inside the neurons and were detected in the soma, axons and dendrites of the neuron (Fig. 3e-f).

\section{Role of Miro-1 in mitochondrial transfer from astrocytes to neurons}

We next assessed the contribution of Miro-1, a RhoGTPase mitochondrial adaptor protein, to mitochondrial transfer from astrocytes to damaged neurons. Astrocytes were transfected with short interfering RNA (siRNA) to knock down Miro-1 (astrocytes ${ }^{\text {Miro-1 siRNA }}$ ) and mitochondria in astrocytes were labeled with mito-GFP. Miro-1 siRNA decreased astrocytic Miro-1 by approximately 50\% (Fig. 4 inset). Neurons were treated with cisplatin and co-cultured with astrocytes ${ }^{\text {Miro-1 }}$ siRNA or astrocytes ${ }^{\text {Scr }}$ siRNA as a control and mitochondrial transfer was quantified. The results in Fig. 4 show that knockdown of Miro-1 


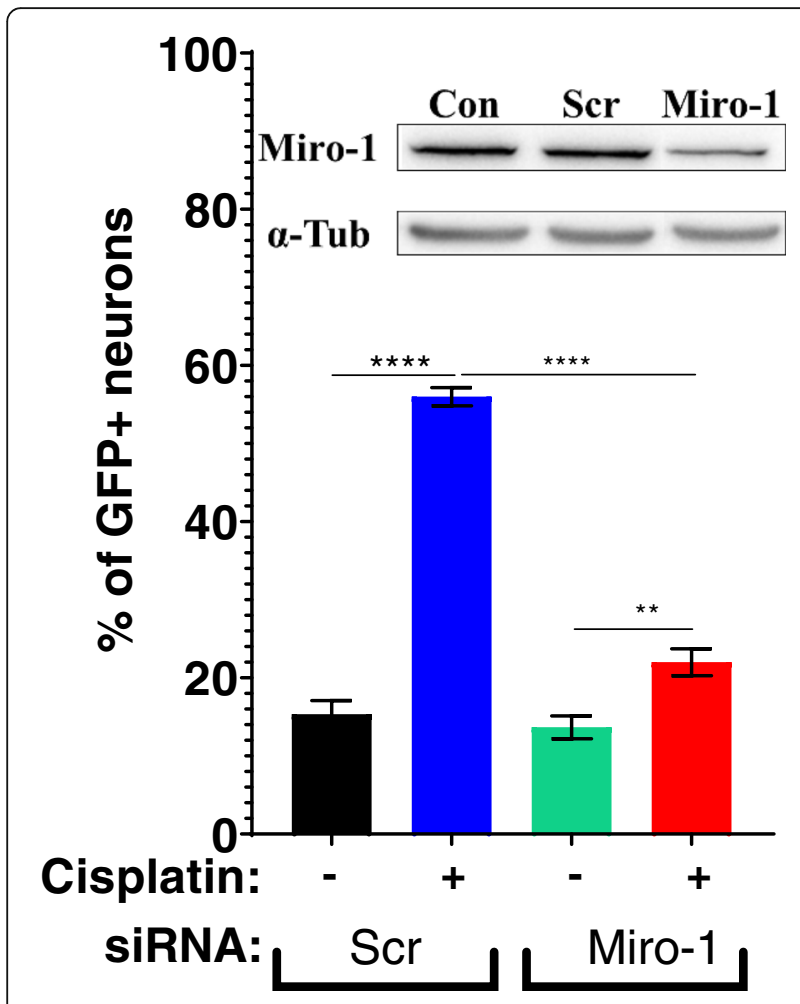

Fig. 4 Role of Miro-1 in mitochondrial transfer from astrocytes to neurons. Astrocytes were transfected with mito-GFP and Miro-1 siRNA or control scrambled (Scr) siRNA cultured with or without cisplatin $24 \mathrm{~h}$ and co-cultured for $17 \mathrm{~h}$ with neurons treated with or without cisplatin. Using confocal microscopy the percentage of neurons containing GFP was quantified. Data represents the mean \pm SEM of 3 independent experiments performed in duplicate. Inset: Western blot to confirm knockdown of Miro-1 in astrocytes after transfecting with mito-GFP, Scr and Miro-1 siRNA. The percentage of knockdown was normalized to the control and quantified. Two-way ANOVA cisplatin $x$ transfected astrocyte interaction: ${ }^{* * *} p<0.0001$, followed by Tukey's post-hoc test: ${ }^{* *} p<0.01,{ }^{* * * *} p<0.0001$

in astrocytes decreased mitochondrial transfer to neurons in comparison to astrocytes treated with control scrambled siRNA (Fig. 4). These findings indicate that Miro-1 plays a key role in the transfer of mitochondria from astrocytes to neurons damaged by cisplatin.

\section{Effects of cisplatin and astrocytes on neuronal calcium dynamics}

We next asked the question whether cisplatin alters neuronal $\mathrm{Ca}^{2+}$ levels and whether co-culture with astrocytes affects cisplatin-induced changes in neuronal calcium dynamics. Resting $\left[\mathrm{Ca}^{2+}{ }_{\mathrm{i}}\right]$ levels, as measured using the calcium indicator Fura-2, were significantly higher in cisplatin-treated neurons than in control neurons (Fig. 5a and b). Co-culture with astrocytes normalized resting $\left[\mathrm{Ca}^{2+}{ }_{\mathrm{i}}\right]$ levels in cisplatin-treated neurons (Fig. 5a and b). The increase in neuronal $\left[\mathrm{Ca}^{2+}{ }_{\mathrm{i}}\right]$ level in response to $20 \mathrm{mM} \mathrm{KCl}$ was smaller in cisplatin-treated than in control neurons and this was also normalized by co-culture with astrocytes, indicating that astrocytes normalize calcium dynamics in neurons treated with cisplatin. (Fig. $5 \mathrm{c}$ and d).

Treatment of neurons with cisplatin increased the time to reach $80 \%$ clearance of $\left[\mathrm{Ca}^{2+}{ }_{\mathrm{i}}\right]$ after exposure to 20 $\mathrm{mM} \mathrm{KCl} \mathrm{(Fig.} \mathrm{5e} \mathrm{and} \mathrm{f),} \mathrm{even} \mathrm{though} \mathrm{maximal} \mathrm{calcium}$ levels were significantly lower in cisplatin-treated neurons (Fig. 5a and b). These findings indicate $\mathrm{Ca}^{2+}$ clearance is impaired in cisplatin treated neurons. Co-culture with astrocytes increased the rate of calcium clearance in cisplatin-treated neurons to a level that was even higher than that of control neurons (Fig. 5e and f).

\section{Mitochondrial transfer underlies the beneficial effects of astrocytes on calcium dynamics in damaged neurons}

To address the question of whether transfer of mitochondria from astrocytes to neurons contributes to the improved neuronal $\mathrm{Ca}^{2+}$ dynamics, we compared calcium responses to $20 \mathrm{mM} \mathrm{KCl}$ of neurons that did or did not contain mCherry+ mitochondria on the same coverslip. As shown in Fig. 6a neurons that contained astrocyte-derived mitochondria showed a larger increase in $20 \mathrm{mM} \mathrm{KCl}$ evoked $\left[\mathrm{Ca}^{2+}{ }_{\mathrm{i}}\right]$ in comparison to neurons in the same culture that did not receive astrocytic mitochondria (Fig. 6a). This finding indicates that transfer of mitochondria from astrocytes to neurons plays a substantial role in restoring the $\mathrm{KCl}$ evoked $\left[\mathrm{Ca}^{2+}{ }_{i}\right]$ increase in neurons damaged by cisplatin.

To further address the contribution of mitochondrial transfer to the normalization of calcium dynamics in neurons in response to co-culture with astrocytes, we assessed the effect of astrocytic Miro-1 knockdown. The results in Fig. $6 \mathrm{~b}$ and $\mathrm{c}$ show that basal calcium levels were lower in cisplatin-damaged neurons co-cultured with control astrocytes ${ }^{\text {Scr siRNA }}$ as compared to astrocy-

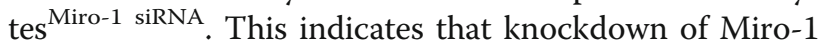
in astrocytes prevented the normalization of baseline calcium levels in cisplatin-treated neurons (Fig. $6 \mathrm{~b}$ and c). Similarly, co-culture of cisplatin-treated neurons with astrocytes $^{\text {Miro-1 siRNA }}$ failed to normalize the $20 \mathrm{mM} \mathrm{KCl}$ evoked $\left[\mathrm{Ca}^{2+}{ }_{i}\right]$ that was observed in the presence of control astrocytes ${ }^{\text {Scr siRNA }}$ (Fig. $6 \mathrm{~d}$ and e). This indicates that the restorative effects of astrocytes on calcium dynamics in cisplatin-treated neurons are abrogated by impairing mitochondrial transfer from astrocytes.

\section{Discussion}

Our in vitro findings suggest that astrocytic mitochondrial transfer to neurons may represent an endogenous repair mechanism to counteract the neurotoxic effects of cisplatin treatment. Our evidence indicates that cisplatin reduces neuronal survival and decreases mitochondrial membrane potential in the surviving neurons. We also 

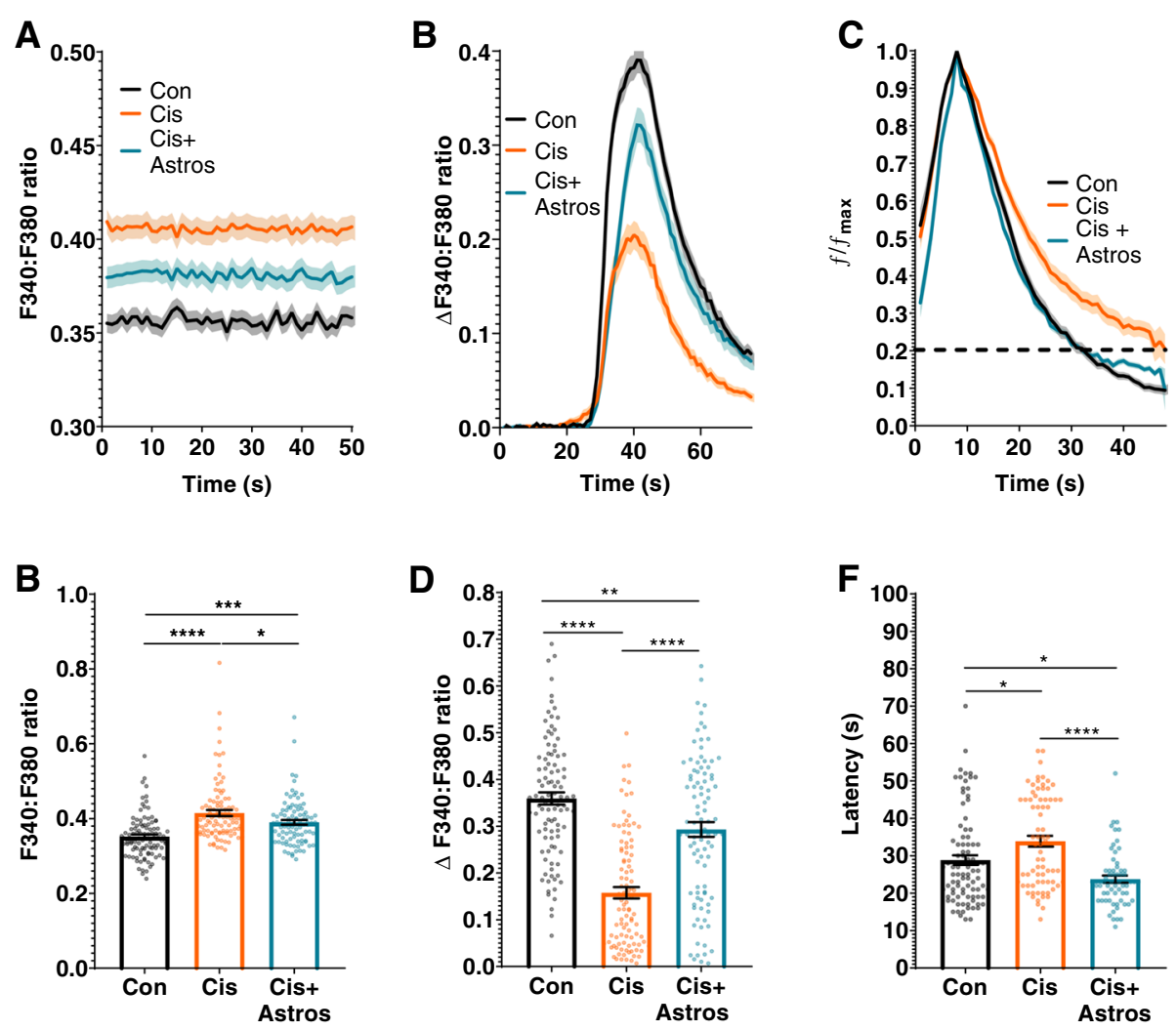

Fig. 5 Effects of cisplatin and astrocytes on neuronal calcium dynamics. Neurons were treated with cisplatin followed by co-culture with astrocytes and calcium dynamics were monitored with Fura-2. a, b. Resting ratio of 340:380 Fura-2 fluorescence intensity (F340:F380) as an indicator of intracellular levels of $\mathrm{Ca}^{2+}$ of neurons treated with or without cisplatin followed by co-culture with or without astrocytes. a. Mean traces and SEM of the F340:F380 ratio of Fura-2 at baseline during first $50 \mathrm{~s}$ of recording. b. Mean F340:F380 ratio during $50 \mathrm{~s}$ of recording for each neuron at rest. Data represents the mean \pm SEM of $>90$ neurons total per group collected in 3 independent experiments. One-way ANOVA, followed by Tukey's post-hoc test: $\left({ }^{*} p<0.05,{ }^{* *} p<0.001,{ }^{* * *} p<0.0001\right)$. c. Mean traces and SEM of the increase in F340:F380 ratio of Fura-2 of neurons responding to stimulation with $20 \mathrm{mM} \mathrm{KCl}$. The threshold change in Fura-2 F340:F380 ratio to be considered a response to $20 \mathrm{mM} \mathrm{KCl}$ was set at an increase of $>5 \times$ standard deviations above baseline average. This excluded $9 \%$ of neurons in the control group, $23 \%$ of neurons in the cisplatin group and $11 \%$ of neurons in the cisplatin + astrocyte group. Data represents the mean \pm SEM of 3 independent experiments with $>70$ neurons for each group. $\mathbf{d}$. Mean change in F340:F380ratio in response to stimulation of neurons with $20 \mathrm{mM} \mathrm{KCl}$. The increase in Fura-2 ratio in response to $20 \mathrm{mM} \mathrm{KCl}$ was calculated for each neuron. Data represent the mean \pm SEM of $n>90$ neurons for each group collected in 3 independent experiments. One-way ANOVA, followed by Tukey's post-hoc test: $\left({ }^{* *} p<0.01,{ }^{* *} p<0.001,{ }^{* * *} p<0.0001\right)$. e Normalized KCl responses show a delayed decay of $\mathrm{Ca}^{2+}$ influx in cisplatin-treated neurons vs. control and cisplatin + astrocytes groups. $\mathbf{f}$. Quantification of latency to return to $20 \%$ of maximal $\mathrm{Ca}^{2+}$ (dotted line). Data represents the mean \pm SEM of 3 independent experiments with $>70$ neurons for each group. One-way ANOVA, followed by Tukey's post-hoc test: $\left({ }^{*} p<0.05,{ }^{* * *} p<0.0001\right)$

show that co-culture of cisplatin-treated neurons with astrocytes results in mitochondrial transfer from astrocytes to neurons and this is associated with normalization of survival and mitochondrial membrane potential. Cisplatin altered neuronal $\mathrm{Ca}^{2+}{ }_{i}$ levels in cortical neurons, and addition of astrocytes normalized neuronal $\mathrm{Ca}^{2+}{ }_{\mathrm{i}}$. Calcium levels were specifically restored in those cisplatin-treated neurons that had received astrocytic mitochondria. Moreover, we show that the Rho-GTPase Miro-1 is essential for the transfer of mitochondria from astrocytes to neurons. SiRNA-mediated knockdown of astrocytic Miro-1 prevented transfer of mitochondria from astrocytes to damaged neurons and prevented the restoration of calcium dynamics in neurons damaged by cisplatin. Collectively, our data support the hypothesis that astrocytes counteract the neurotoxic effects of cisplatin by transfering mitochondria to neurons damaged by cisplatin via a Miro-1dependent pathway.

The effects of astrocytic mitochondrial transfer have been previously shown in disease models of Alexander's disease and ischemic stroke [36, 37]. In our in vitro system, we investigated whether astrocytes could also have a restorative effect on neuronal damage as a result of cisplatin treatment. To that end we pre-incubated neurons with cisplatin which caused a significant decrease in neuronal survival and a reduction in the mitochondrial membrane potential in the surviving neurons, and subsequently co-cultured the surviving neurons with 

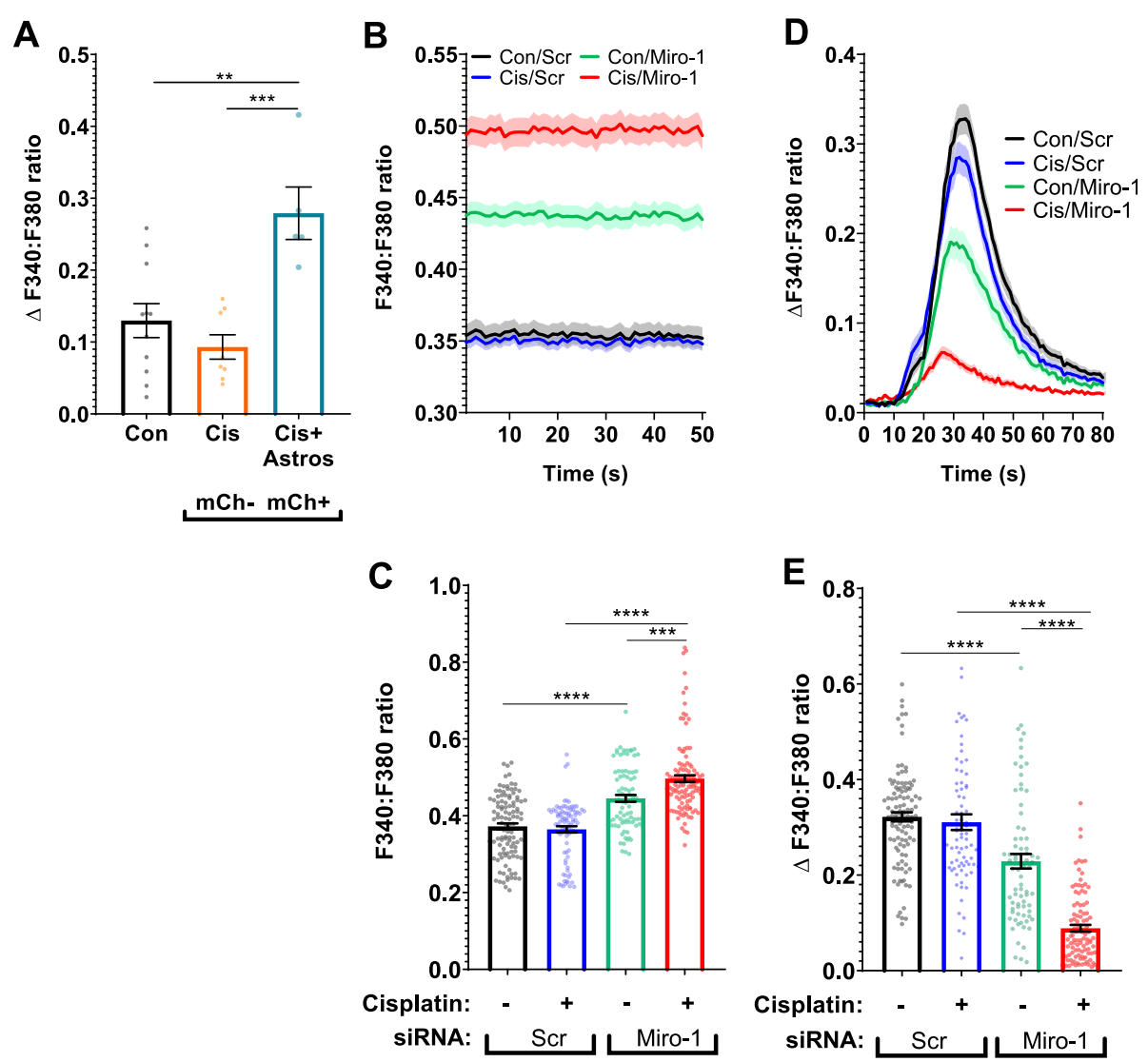

Fig. 6 Mitochondrial transfer underlies the beneficial effects of astrocytes on calcium dynamics in neurons damaged by cisplatin. a. Neurons were treated with and without cisplatin for $24 \mathrm{~h}$ and co-cultured with astrocytes transfected with mito-mCherry (labeled $\mathrm{mCh}$ ) for $17 \mathrm{~h}$. The calcium response to $20 \mathrm{mM} \mathrm{KCl}$ was compared in cisplatin-treated neurons cultured without astrocytes (black bar), or with astrocytes separated into mCherry negative neurons (orange bar; do not contain astrocytic mitochondria) and mCherry positive neurons (blue bar; contain astrocytederived mitochondria). The mean increase in the F340:F380 ratio of Fura-2 fluorescence in response to $20 \mathrm{mM} \mathrm{KCl}$ was calculated as in Fig. 5. Data represents the mean \pm SEM of 3 independent experiments with $n=24$ neurons in total. One-way ANOVA, followed by Tukey's post-hoc test: $\left({ }^{* *} p<0.01,{ }^{* * *} p<0.0001\right)$. b. Resting F340:F380 ratio of Fura-2 of neurons treated with cisplatin followed by co-culture with astrocytes transfected with mito-GFP and scr siRNA or Miro-1 siRNA. c. Mean F340:F380 ratio was calculated for each neuron for first $50 \mathrm{~s}$ and data represents the mean \pm SEM of 3 independent experiments with $n>80$ neurons for each group. Two-way ANOVA: cisplatin $x$ transfected astrocyte interaction: ${ }^{* * *} p<0.001$ followed by Tukey's post-hoc test: ${ }^{* * *} p<0.001,{ }^{* * *} p<0.0001$ ). d. Mean traces and SEM of the F340:F380 ratio in response to $20 \mathrm{mM}$ $\mathrm{KCl}$ stimulus in neurons treated with and without cisplatin and co-cultured with astrocytes transfected with scrambled (Scr) or Miro-1 siRNA. The mean F340:F380 ratio KCl responses were normalized to baseline. e. The change in F340:F380 ratio between baseline and maximum peak value from the $20 \mathrm{mM} \mathrm{KCl}$ stimulus was calculated which correlated with $\left[\mathrm{Ca}^{2+}\right]_{\mathrm{i}}$ increase in neurons. Mean increase in F340:F380 ratio ratio was calculated for each neuron for $3 \mathrm{~s}$ before $20 \mathrm{mM} \mathrm{KCl}$ and subtracted from mean F340:F380 ratio at the maximum response to $20 \mathrm{mM} \mathrm{KCl}$. Data represents the mean \pm SEM of 3 independent experiments with $n>80$ neurons for each group. Two-way ANOVA: cisplatin $x$ transfected astrocyte interaction: $\left({ }^{* * *} p<0.0001\right)$, followed by Tukey's post-hoc test $\left({ }^{* * *} p<0.0001\right)$

astrocytes. Our data show that astrocytes can repair already existing neuronal damage as a result of cisplatin because co-culture with astrocytes restored neuronal mitochondrial membrane potential and protected against further neuronal death. Platinum compounds directly damage the DNA by forming adducts and this interferes with cell proliferation [38]. It is likely that DNA adducts are also formed in neurons exposed to cisplatin, but because these cells are post-mitotic, it is not known what the effect on cell function will be. It should be noted that we have shown previously that in vivo, preventing mitochondrial damage is sufficient to prevent cognitive deficits that develop in response to treatment with cisplatin [8, 39]. Therefore, we propose that mitochondrial toxicity is a key factor in cisplatin-induced damage to neurons.

A structural transport mechanism that has been shown to be involved in intercellular mitochondrial transfer are tunneling nanotubes (TNTs). TNTs are thin nonadherent actin-rich membranous structures with diameters between 50 and $1500 \mathrm{~nm}$ that can span long distances of several hundred nm [17, 18, 20, 22, 40-42]. These TNT form direct connections between cells to transport cellular components including cytoplasm, ions, lipid droplets, viral and bacterial pathogens, genetic material and organelles 
like lysosomes, and last but not least, mitochondria [17, 43, 44]. Multiple authors have described that the mitochondrial Rho-GTPase-1 protein (Miro-1) is a crucial player in intercellular mitochondrial transfer via TNTs. Miro-1 is an outer mitochondrial membrane protein, that binds to Milton, a kinesin/dynein adaptor protein and this promotes mitochondrial motilit y[26, 28, 45, 46]. We have now expanded this knowledge by showing that decreasing Miro-1 expression in astrocytes decreased the transfer of mitochondria from astrocytes to damaged neurons. Apparently, astrocytic Miro-1 is required for transfer of astrocyte mitochondria to neurons. This could imply that the formation of TNTs for intercellular transport is executed by astrocytes rather than neurons. Jiang et al. have described that the cyclic ADP ribose hydrolase CD-38 plays an important role in astrocytic mitochondrial transfer [47]. Inhibition of CD-38 with apigenin significantly reduced astrocytic mitochondrial transfer and it has been suggested that CD-38 may be involved in TNT formation [48]. Furthermore, in an in vitro myeloma cancer model, myeloma cells were shown to receive mitochondria from non-malignant bone marrow stromal cells through CD38-dependent tumor-derived formation of TNTs [48]. However, we have to keep in mind that this phenomenon could be specific for tumor cells.

Our data shows that astrocytes transfer mitochondria to neurons damaged by cisplatin while there is only very limited transfer under control conditions. Similarly, we have reported previously mesenchymal stem cells transfer more mitohcondria to neuronal stem cells (NSCs) damaged by cisplatin than to control NSCs [20]. Berridge et al. showed that astrocytes transfer more mitochondria to damaged neurons in an ischaemic stroke model in comparison to control [49]. We hypothesize that astrocytes may repond to a "help" signal that is expressed by damaged neurons leading to the transfer of mitochondria. It has been suggested that acculuation of p53 on damaged mitochondrial membranes and/or the release of Damage-Associated Molecular Patterns (DAMPS) could serve as the "help" signal [50-52]. Further studies are needed to identify which signals initiate the transfer process.

Miro-1 modulates mitochondrial shape in response to cytosolic $\mathrm{Ca}^{2+}$ stress, a phenomenon which is distinct from fission and fusion [53]. Additionally, Stephen et al. [27], have shown that Miro-1 positions mitochondria in areas within the astrocytic processes that are near neuronal synaptic activity where high energy and $\mathrm{Ca}^{2+}$ modulation is necessary. With this in mind it could be possible that astrocytic Miro-1 could be important for positioning mitochondria for transfer in response to neuronal $\mathrm{Ca}^{2+}$ changes due to cisplatin damage. It is very well possible that additional activities of astrocytes involved in mitochondrial/cellular health could be of importance as well. However, we specifically observed restoration of $\left[\mathrm{Ca}^{2+}\right]_{\mathrm{i}}$ levels in those neurons that actually had received astrocytic mitochondria which implies that mitochondrial transfer plays a crucial role in the restorative effect of astrocytes on neuronal $\left[\mathrm{Ca}^{2+}{ }_{\mathrm{i}}\right]$ levels and health.

Neuronal $\mathrm{Ca}^{2+}$ levels are tightly regulated and are critical for many processes in neurons including neurotransmission, depolarization, and synaptic activities. Mitochondria play an important role in controlling $\mathrm{Ca}^{2+}$ levels by taking up, buffering and releasing cytosolic $\mathrm{Ca}^{2+}$. Cisplatin can negatively alter $\mathrm{Ca}^{2+}$ levels such as has been observed in the dorsal root ganglia [54]. Our findings expand this knowledge by showing that cisplatin leads to dysfunctional cortical neuronal $\mathrm{Ca}^{2+}$ levels. Abnormalities in resting and $\mathrm{KCl}$-evoked $\mathrm{Ca}^{2+}$ increases and clearance due to treatment of cortical neurons with cisplatin were reversed in the presence of astrocytes. As mitochondria are important for neuronal $\mathrm{Ca}^{2+}$ dynamics we suggest that astrocytic mitochondrial transfer is key to normalizing the neuronal mitochondrial network. It remains to be determined whether the normalization of $\mathrm{Ca}^{2+}$ dynamics results from a direct role of the donated mitochondria in calcium buffering or a secondary effect on other key regulators of calcium homeostatis such as the endoplasmic reticulum (ER), Golgi apparatus, and peroxisomes, or the functioning of ion channels and pumps [55-59]. However, our findings do show that transfer of mitochondria normalizes neuronal $\mathrm{Ca}^{2+}$ dynamics in neurons damaged by cisplatin.

Our cell survival studies showed that astrocytes are much less sensitive to cisplatin than neurons. RNA-seq data [60], show that astrocytes have a higher concentration of the mitochondrial polymerase gamma (poly) in comparison to neurons which is the sole polymerase involved in mitochondrial replication, mutagenesis and repair of mtDNA. Therefore, we suggest that the releatively high activity of poly in astrocytes may lead to efficient repair of cisplatin adducts which could contribute to the observed astrocytic resiliency. In addition, astrocytes have a higher concentration of the copper tansporters ATP7a and ATP7b in comparison to neurons and other glial cells [60]. ATP7a and ATP7b are copper transporters that also promote platinum efflux and thereby may contribute to cellular resistance to cisplatin [61-63].

An important translational question still lingers: if astrocytic mitochondrial transfer occurs in the brain after cisplatin treatment, why do patients undergoing chemotherapy still experience neurotoxicity leading to chemotherapy-induced cognitive impairment? One argument could be that the endogenous restorative capacity of astrocytes is no longer sufficient when patients are treated for a long time, which is common for chemotherapy with cisplatin. Indeed, the risk of 
developing chemobrain increases with duration of treatment [10, 64-67]. Moreover, our preliminary data indicate that exposure of mice to a single round of cisplatin treatment does not induce cognitive deficits, whereas two rounds of cisplatin do induce significant decreases in performance in tests of cognitive function $[8,68]$. Although astrocytes may still prevent the actual death of (non self-renewing) adult neurons, they may may fail to completely restore mitochondrial health. The endogenous protective activity of transferring healthy mitochondria from astrocytes to damaged neurons may also become less efficient in the aging brain. From the literature it is known that the severity of the behavioral neurotoxic effects are correlated with age $[65,66,69]$. When endogenous protective mechanisms are not sufficient, interventions aimed at restoring mitochondrial health may provide additional help. Indeed, we showed recently that cell therapy with mesenchymal stem cells or a pharmacolgocial intervention with PFT- $\mu$ and HDAC6 inhibitor both reverse cisplatin-induced neuronal mitochondrial abnomarlities as well as cognitive impairment in mice $[8,11,28,30$, $39,51,70]$.

In conclusion, we propose that astrocytic mitochondrial transfer is an important endogenous protection mechanism against chemotherapy neurotoxicity. Promoting astrocytic mitochondrial transfer could represent interesting therapeutic targets to prevent or treat the devastating effects of chemotherapy on the brain.

\section{Conclusions}

This novel study demonstrates that: 1) Astrocytes rescue survival and function of neurons damaged by cisplatin by donating their mitochondria to the more vulnerable cortical neurons. 2) Mechanistically, we show that cisplatin depolarizes neuronal mitochondrial membrane potential without decreasing neuronal mitochondrial content. Astrocytes restore neuronal mitochondrial membrane potential leading to an increase in mitochondrial content. 3) Miro-1 siRNA decreases mitochondrial transfer from astrocytes to neurons indicating that Miro-1 is involved in astrocytic mitochondrial transfer. 4) Cisplatin increased resting $\left[\mathrm{Ca}^{2+}{ }_{\mathrm{i}}\right]$ levels in neurons and impaired neuronal calcium clearance. Astrocytes normalized neuronal resting $\left[\mathrm{Ca}^{2+}{ }_{\mathrm{i}}\right]$ levels and improved neuronal calcium clearance. 5) To investigate that the transfer of mitochondria is crucial for the rescue of neuronal function, we show here that those neurons that contained astrocyte-derived mitochondria had improved response to $20 \mathrm{mM} \mathrm{KCl}$ in comparison to neurons that did not contain astrocyte-derived mitochondria. Astrocytes transfected with Miro-1 siRNA, which decreases mitochondrial transfer from astrocytes to neurons, failed to prevent the normalization of baseline calcium levels and response to $20 \mathrm{mM} \mathrm{KCl}$ in neurons damaged by cisplatin. In conclusion this data illustrates that astrocytes transfer mitochondria to neurons damaged by cisplatin and restores neuronal calcium dynamics, survival, mitochondrial membrane potential, and increases mitochondrial content. Our findings contribute to further understanding of endogenous protective neuro-glial mechanisms that aid in protection of the brain against the devastating effects of chemotherapy on healthy cells that are vulnerable to cancer treatment.

\section{Abbreviations}

$\left[\mathrm{Ca}^{2+}{ }_{\mathrm{i}}\right]$ : Intracellular calcium concentration; $\mathrm{Ca}^{2+}$ : Calcium; CTB: CellTracker Blue; CTDR: CellTracker Deep Red; CTG: CellTracker Green; F340:F380: ratio of fluorescence at 340 and $380 \mathrm{~nm}$; FCCP: Carbonilcyanide p-

triflouromethoxyphenylhydrazone; KCl: Potassium chloride; Miro-

1: Mitochondrial Rho GTPase 1, also known as Rho-1; Mito-GFP: GFP coupled to a mitochondrial localization sequence; Mito-mCherry: mCherry coupled to a mitochondrial localization sequence; siRNA: Short interfering RNA;

TMRM: Tetramethylrhodamine; TNTs: Tunneling nanotubes

\section{Acknowledgements}

Not applicable.

\section{Authors' contributions}

KE proposed and designed the study. KE acquired all the data and interpreted the data. KE also wrote and revised the current manuscript. AS substantially contributed to designing experiments to measure neuronal calcium in the presence of cisplatin and astrocytes. AS substantially contributed to acquisition of neuronal calcium data and analysis of the calcium data. AS was also a major contributor to writing and revising the manuscript. NU contributed to designing the study by providing cortical neurons and astrocytes, along with providing instructions on how to isolate cortical cells. RT performed and analyzed the western blots for Miro-1 siRNA and Scrambled siRNA knockdown in astrocytes. AK significantly contributed to conceptualizing and designing all steps of the study, and significantly contributed to interpreting the data. AK was a major contributor to writing and revising the manuscript. $\mathrm{CH}$ provided funding for the study, and significantly contributed to the conception and design of the study. $\mathrm{CH}$ significantly contributed to interpreting the data, and significantly contributed to writing and revising the manuscript. All authors read and approved the final manuscript.

\section{Funding}

This study was supported by grants RO1 CA208371 (CJH, AK) and R01 CA227064 (AK, CJH) from the National Cancer Institute. This work was also supported by the $\mathrm{NIH} / \mathrm{NCl}$ under award number P30CA016672 to MD Anderson Cancer Center.

\section{Availability of data and materials}

The datasets used and/or analyzed during the current study are available from the corresponding author on reasonable request.

Ethics approval and consent to participate

Not applicable.

\section{Consent for publication}

Not applicable.

\section{Competing interests}

The authors declare that they have no competing interests.

\section{Author details}

${ }^{1}$ Division of Internal Medicine, Department of Symptom Research, Laboratories of Neuroimmunology, The University of Texas MD Anderson Cancer Center, Houston, TX 77030, USA. ${ }^{2}$ Department of Neurobiology \& 
Anatomy, The University of Texas McGovern Medical School, Houston, TX 77030, USA. ${ }^{3}$ Department of Neurology, The University of Texas McGovern Medical School, Houston, TX 77030, USA.

Received: 8 January 2020 Accepted: 13 February 2020

Published online: 20 March 2020

\section{References}

1. Kann O, Kovács R (2007) Mitochondria and neuronal activity. Am J Physiol Physiol 292(2):C641-C657

2. Burté F, Carelli V, Chinnery PF, Yu-Wai-Man P (2015) Disturbed mitochondrial dynamics and neurodegenerative disorders. Nat Rev Neurol 11(1):11-24

3. Flippo KH, Strack S (2017) Mitochondrial dynamics in neuronal injury, development and plasticity. J Cell Sci 130(4):671-681

4. McCue HV, Haynes LP, Burgoyne RD (2010) The diversity of calcium sensor proteins in the regulation of neuronal function. Cold Spring Harb Perspect Biol 2(8):a004085

5. Pivovarova NB, Andrews SB (2010) Calcium-dependent mitochondrial function and dysfunction in neurons. FEBS J 277(18):3622-3636

6. Guo L, Tian J, Du H (2017) Mitochondrial dysfunction and synaptic transmission failure in Alzheimer's disease. J Alzheimers Dis 57(4):1071-1086

7. Smith GM, Gallo G (2018) The role of mitochondria in axon development and regeneration. Dev Neurobiol 78(3):221-237

8. Chiu GS et al (2017) Pifithrin-m prevents cisplatin-induced chemobrain by preserving neuronal mitochondrial function. Cancer Res 77(3):742-752

9. Devine MJ, Kittler JT (2018) Mitochondria at the neuronal presynapse in health and disease. Nat Rev Neurosci 19(2):63-80

10. Ren $X$ et al (2019) The triangle of death of neurons: oxidative damage, mitochondrial dysfunction, and loss of choline-containing biomolecules in brains of mice treated with doxorubicin. Advanced insights into mechanisms of chemotherapy induced cognitive impairment ('chemobrain') involving TNF-a. Free Radic Biol Med 134:1-8

11. Chiu GS et al (2018) Nasal administration of mesenchymal stem cells restores cisplatin-induced cognitive impairment and brain damage in mice. Oncotarget 9(85):35581-35597

12. Bayraktar OA, Fuentealba LC, Alvarez-Buylla A, Rowitch DH (2014) Astrocyte development and heterogeneity. Cold Spring Harb Perspect Biol 7(1): a020362

13. Bazargani N, Attwell D (2016) Astrocyte calcium signaling: The third wave. Nat Neurosci 19(2):182-189 Nature Publishing Group

14. Bozoyan L, Khlghatyan J, Saghatelyan A (2012) Astrocytes control the development of the migration-promoting vasculature scaffold in the postnatal brain via VEGF signaling. J Neurosci 32(5):1687-1704

15. Gengatharan A, Bammann RR, Saghatelyan A (2016) The role of astrocytes in the generation, migration, and integration of new neurons in the adult olfactory bulb. Front Neurosci 10:149

16. Lundgaard I, Osório MJ, Kress BT, Sanggaard S, Nedergaard M (2014) White matter astrocytes in health and disease. Neuroscience 276:161-173

17. Vignais ML, Caicedo A, Brondello JM, Jorgensen C (2017) Cell connections by tunneling nanotubes: Effects of mitochondrial trafficking on target cell metabolism, homeostasis, and response to therapy, vol 2017. Stem Cells International

18. Wang $Y$, Cui J, Sun $X$, Zhang $Y$ (2011) Tunneling-nanotube development in astrocytes depends on p53 activation. Cell Death Differ 18(4):732-742

19. Davis CO et al (2014) Transcellular degradation of axonal mitochondria. Proc Natl Acad Sci U S A 111(26):9633-9638

20. Boukelmoune N, Chiu GS, Kavelaars A, Heijnen CJ (2018) Mitochondrial transfer from mesenchymal stem cells to neural stem cells protects against the neurotoxic effects of cisplatin. Acta Neuropathol Commun 6(1):139

21. Caicedo A et al (2015) MitoCeption as a new tool to assess the effects of mesenchymal stem/stromal cell mitochondria on cancer cell metabolism and function. Sci Rep 5:9073

22. Babenko VA et al (2018) Mirol enhances mitochondria transfer from multipotent mesenchymal stem cells (MMSC) to neural cells and improves the efficacy of cell recovery. Molecules 23(3)

23. Rogers RS, Bhattacharya J (2013) When Cells Become Organelle Donors. Physiology 28(6):414-422

24. Jackson JG, Robinson MB (2015) Reciprocal regulation of mitochondrial dynamics and calcium signaling in astrocyte processes. J Neurosci 35(45): 15199-15213
25. Oeding SJ et al (2018) Identification of Miro1 and Miro2 as mitochondrial receptors for myosin XIX. J Cell Sci 131(17)

26. Schwarz TL (2013) Mitochondrial trafficking in neurons. Cold Spring Harb Perspect Med 5(6)

27. Stephen TL et al (2015) Miro1 regulates activity-driven positioning of mitochondria within astrocytic processes apposed to synapses to regulate intracellular calcium signaling. J Neurosci 35(48):15996-16011

28. Kalinski AL et al (2019) Deacetylation of Miro1 by HDAC6 blocks mitochondrial transport and mediates axon growth inhibition. J Cell Biol 218(6):1871-1890

29. Ahmad T et al (2014) Miro1 regulates intercellular mitochondrial transport \& enhances mesenchymal stem cell rescue efficacy. EMBO J 33(9):994-1010

30. Ma J, Huo XJ, Jarpe MB, Kavelaars A, Heijnen CJ (2018) Pharmacological inhibition of HDAC6 reverses cognitive impairment and tau pathology as a result of cisplatin treatment. Acta Neuropathol Commun 6(1):103

31. Shirihai OS, Song M, Dorn GW (2015) How mitochondrial dynamism orchestrates mitophagy. Circ Res 116(11):1835-1849

32. Mattson MP, Gleichmann M, Cheng A (2018) Mitochondria in Neuroplasticity and Neurological Disorders. Neuron 60:748-766

33. Andres AL, Gong X, Di K, Bota DA (2014) Low-doses of cisplatin injure hippocampal synapses: a mechanism for 'chemo' brain? Exp Neurol 255:137-144

34. Rego AC, Vesce S, Nicholls DG (2001) The mechanism of mitochondrial membrane potential retention following release of cytochrome $\mathrm{c}$ in apoptotic GT1-7 neural cells. Cell Death Differ 8(10):995-1003

35. Shepherd AJ et al (2018) Angiotensin II triggers peripheral macrophage-tosensory neuron redox crosstalk to elicit pain. J Neurosci 38(32):7032-7057

36. Gao L, Zhang Z, Lu J, Pei G (2019) Mitochondria are dynamically transferring between human neural cells and Alexander disease-associated GFAP mutations impair the Astrocytic transfer. Front Cell Neurosci 13:316

37. Hayakawa K et al (2016) Transfer of mitochondria from astrocytes to neurons after stroke. Nature 535(7613):551-555

38. Roos WP, Kaina B (2013) DNA damage-induced cell death: from specific DNA lesions to the DNA damage response and apoptosis. Cancer Lett 332: 237-248

39. Maj MA, Ma J, Krukowski KN, Kavelaars A, Heijnen CJ (2017) Inhibition of mitochondrial p53 accumulation by PFT- $\mu$ prevents cisplatin-induced peripheral neuropathy. Front Mol Neurosci 10:108

40. Gousset $\mathrm{K}$ et al (2013) Myo10 is a key regulator of TNT formation in neuronal cells. J Cell Sci 126(Pt 19):4424-4435

41. Las G, Shirihai OS (May 2014) Miro1: new wheels for transferring mitochondria. EMBO J 33(9):939-941

42. Abounit S, Delage E, Zurzolo C, Abounit S, Delage E, Zurzolo C (2015) Identification and characterization of tunneling nanotubes for intercellular trafficking. In: Current protocols in cell biology. Wiley, Hoboken, 67(1): 12.10 $1-12.10 .21$

43. Li CJ, Chen PK, Sun LY, Pang CY (2017) Enhancement of Mitochondrial Transfer by Antioxidants in Human Mesenchymal Stem Cells. Oxidative Med Cell Longev 2017:8510805

44. Astanina K et al (2015) Lipid droplets as a novel cargo of tunnelling nanotubes in endothelial cells. Sci Rep 5:11453

45. Lee KS, Lu B (2014) The myriad roles of miro in the nervous system: Axonal transport of mitochondria and beyond. Front Cell Neurosci (8, October. Frontiers Media S.A.)

46. Melkov A, Baskar R, Alcalay Y, Abdu U (2016) A new mode of mitochondrial transport and polarized sorting regulated by dynein, Milton and Miro. Development 143(22):4203-4213

47. Jiang $D$ et al (2016) Mitochondrial transfer of mesenchymal stem cells effectively protects corneal epithelial cells from mitochondrial damage. Cell Death Dis $7(11)$

48. Marlein CR et al (2019) CD38-driven mitochondrial trafficking promotes bioenergetic plasticity in multiple myeloma. Cancer Res 79(9):2285-2297

49. Berridge MV, Schneider RT, McConnell MJ (2016) Mitochondrial Transfer from Astrocytes to Neurons following Ischemic Insult: Guilt by Association? Cell Metab 24(3):376-378

50. Wang Y, Cui J, Sun X, Zhang Y (2011) Tunneling-nanotube development in astrocytes depends on p53 activation. Cell Death Differ 18(4):732-742

51. Vichaya EG et al (2015) Mechanisms of chemotherapy-induced behavioral toxicities. Front Neurosci 9:131

52. Wilkins HM, Weidling IW, Ji Y, Swerdlow RH (2017) Mitochondria-derived damage-associated molecular patterns in neurodegeneration. Front Immunol 8:508 
53. Nemani $\mathrm{N}$ et al (2018) MIRO-1 determines mitochondrial shape transition upon GPCR activation and Ca2+ stress. Cell Rep 23(4):1005-1019

54. Leo M, Schmitt L-I, Jastrow H, Thomale J, Kleinschnitz C, Hagenacker T (2017) Cisplatin alters the function and expression of N-type voltage-gated calcium channels in the absence of morphological damage of sensory neurons. Mol Pain 13:1744806917746565

55. Bernocchi $\mathrm{G}$ et al (2015) Neurotoxic effects of platinum compounds: studies in vivo on intracellular calcium homeostasis in the immature central nervous system. Toxics 3(2):224-248

56. Prins D, Michalak M (2011) Organellar calcium buffers. Cold Spring Harb Perspect Biol 3(3):1-16

57. Modi S et al (2019) Miro clusters regulate ER-mitochondria contact sites and link cristae organization to the mitochondrial transport machinery. Nat Commun 10(1):4399

58. Luo Y, Bond JD, Ingram VM (1997) Compromised mitochondrial function leads to increased cytosolic calcium and to activation of MAP kinases. Proc Natl Acad Sci U S A 94(18):9705-9710

59. Krebs J, Agellon LB, Michalak M (2015) Ca2+ homeostasis and endoplasmic reticulum (ER) stress: An integrated view of calcium signaling. Biochem Biophys Res Commun 460(1):114-121

60. "Brain RNA-Seq." [Online]. Available: http://www.brainrnaseq.org/. [Accessed 27 Nov 2019]

61. Kilari D, Guancial E, Kim ES (2016) Role of copper transporters in platinum resistance. World J Clin Oncol 7(1):106-113

62. Kuo MT, Chen HHW, Song IS, Savaraj N, Ishikawa T (2007) The roles of copper transporters in cisplatin resistance. Cancer Metastasis Rev 26(1): 71-83

63. Blair BG, Larson C, Safaei R, Howell SB (2009) Copper transporter 2 regulates the cellular accumulation and cytotoxicity of cisplatin and carboplatin. Clin Cancer Res 15(13):4312-4321

64. Wang XM, Walitt B, Saligan L, Tiwari AFY, Cheung CW, Zhang ZJ (2015) Chemobrain: A critical review and causal hypothesis of link between cytokines and epigenetic reprogramming associated with chemotherapy. Cytokine 72(1):86-96

65. Gutmann DH (2019) Clearing the Fog surrounding Chemobrain. Cell 176(12):2-4

66. Asher A (2011) Cognitive dysfunction among Cancer survivors. Am J Phys Med Rehabil 90(Suppl 1):S16-S26

67. Jiang T, Cadenas E (2014) Astrocytic metabolic and inflammatory changes as a function of age. Aging Cell 13(6):1059-1067

68. Zhou W, Kavelaars A, Heijnen CJ (2016) Metformin prevents cisplatininduced cognitive impairment and brain damage in mice. PLoS One 11(3)

69. Chiang ACA, Huo X, Kavelaars A, Heijnen CJ (2019) Chemotherapy accelerates age-related development of tauopathy and results in loss of synaptic integrity and cognitive impairment. Brain Behav Immun 79:319325

70. Van Den Bosch L (2019) HDAC6 and Miro1: Another interaction causing trouble in neurons. J Cell Biol 218(6):1769-1770

\section{Publisher's Note}

Springer Nature remains neutral with regard to jurisdictional claims in published maps and institutional affiliations.

Ready to submit your research? Choose BMC and benefit from:
- fast, convenient online submission
- thorough peer review by experienced researchers in your field
- rapid publication on acceptance
- support for research data, including large and complex data types
- gold Open Access which fosters wider collaboration and increased citations
- maximum visibility for your research: over 100M website views per year
At BMC, research is always in progress.
Learn more biomedcentral.com/submissions

